Gill Scott

Nottingham Trent University

Department of Secondary \& Tertiary Education

Clifton Lane

Nottingham

NG11 8 NJ

Tel: $\quad 01158483117$

e-mail: $\quad$ gill.scott@ntu.ac.uk 


\title{
Caseloading and the changing roles of the lecturer and manager
}

\begin{abstract}
Caseloading is a term that has been around in Further Education since colleges were made independent of Local Education authorities in 1993. It is however a term that is widely mis-understood. Its use raises mixed emotions in people. Managers see it potentially as a tool to get more work out of the same number of, or fewer people. Unions and staff seem to view it in a similar way, and therefore tend to resist it. This paper describes the predominant Caseloading models and the political, economic and curriculum pressures that brought the concept to the fore.
\end{abstract}

At the same time as Caseloading has been developed as a management model in some colleges lecturer contracts, role and employment patterns have been changing. A major element of this has been the introduction of "Teaching Support" staff into the curriculum delivery team. The introduction of these types of post has potentially wide ranging implications for the role of lecturers in the future.

In addition the role of the manager continues to develop, with increased accountability for budgets, targets, staff and student management. Caseloading also has implications for the role and scope of senior and middle managers and the devolution of authority within a college. 
In the light of these implications for lecturers and managers the reasons why Caseloading has not been, and is unlikely to be, widely used are explored. 


\section{Caseloading and the changing role of the lecturer and manager}

\section{What is Caseloading?}

Caseloading is a term that has been around in Further Education since colleges were made independent of Local Education authorities in 1993. It is however a term that is widely mis-understood. Its use raises mixed emotions in people. Managers see it potentially as a tool to get more work out of the same number of, or fewer people. Unions and staff seem to view it in a similar way, and therefore tend to resist it. There is a general conception that Caseloading is about individual workloads and weightings for types of activities, following a social services type model, where staff have a "caseload" of clients. This, however, is only part of the Caseloading model. The full Caseloading model is based on individuals or teams, with output targets related to their student recruitment, and the devolution of the resources to achieve those outputs.

The move towards "student centred learning" and away from traditional, closely structured programmes has generated a need to change staffing processes in Further Education and Caseloading is seen as one way of achieving this (Kedney and Scribbins 1995b). It is also seen as a practical method of utilising resources effectively (Sallis 1996),

With fewer student timetabled hours and a move towards resource based learning in all areas the closely defined role of the lecturer has to develop to 
match the changes in the experience of the student. Lecturer contracts that limit contact time and place restrictions on flexible ways of working place barriers to implementation of flexible delivery models. In some colleges Caseloading is seen as a way of avoiding the restrictions of the lecturers "Silver Book" contract. It was in attempting to remove the restrictions inherent within this nationally negotiated contract that the first impetus for Caseloading was developed. Kedney and Scribbins (1995b ) point out that by using a Caseloading model, "the detailed, shared but partial codification of the Silver Book can thus be replaced by a more comprehensive, map covering most or all of the lecturer's duties and contractually available time." (p5)

As most colleges have now moved to more flexible, college negotiated, contracts this is less of an issue in itself. However, restrictions usually remain in defining and controlling the use of lecturer time and much time and effort within colleges is still spent on discussions regarding equity of workload, appropriate activities and the amount of time that can be directed by college managers. Caseloading is an attempt to move the basis of this discussion from a continuing battle between college managers and teachers, to one where the teachers allocate the activities, based upon their professional skill and judgement. The overall level of resources remains within the manager's hands, and the allocation within a Caseloading model must be transparent for it to work; but in essence Caseloading presents a cultural paradigm shift, from one of control and direction to one of devolution and empowerment. However the model adopted must relate to local circumstances. 
Two predominant models of Caseloading have emerged within Further Education.

\section{Workloading}

Workloading aims to balance individual workloads, by recognising the different elements that may make up a teacher's activities. These activities extend beyond "class contact" to tutorial and recruitment activities, preparation and new developments, professional development and marketing. All the various activities are categorised and assigned a weighting factor. These weightings are usually based upon a measure of the complexity of the tasks and the preparation and expertise required to carry them out. Different styles of teaching and assessments will therefore carry different weightings as will activities directly connected with learning, in different settings. A workshop activity, which requires supervision but little preparation or assessment, will therefore have a lower weighting than a formal taught session with detailed theoretical input and related assessment. Numbers of students in a group and the level of the subject will also be reflected in the weighting.

Workloading calculating formulas are used to adjust nominal working time and create weightings and compensations for each activity carried out. All teaching staff will therefore have the same nominal working load. The weighting of activity will adjust the actual workload to reflect the complexity of the tasks being undertaken. 
This system allows for recognition of non-teaching duties, and the different demands of varying teaching duties. It is more sophisticated than the old course levels systems, which graded a course more highly the more advanced the level, and reduced the teaching load accordingly. The system is designed to produce equity of workload and clear management systems for allocating work to staff.

Drawbacks identified with the workload system include the continuing focus on class contact hours and compensation for those hours. This does not break the old culture of hour counting. In addition it can be very complex and bureaucratic, with detailed systems of weightings required and detailed systems of recording. The control of the activities and the weightings remains with management, engendering a control attitude. The responsibility and ownership of the activities is not placed with the lecturers. The system also starts from a base of measuring work. In an atmosphere of tension, where everyone feels that they are the one who is working hardest, everyone will seek to have an actual reduction in workload through this system. In a time of continuing efficiency gains the opposite is the objective of managers. The workload system can therefore feed feelings of distrust and conflict and entrench both sides in time consuming disputes over weightings and relative values.

The Workload system, in my view, does not achieve the improvements in flexibility, ownership and resource utilisation that are being sought from Caseloading. In some instances where it has been tried the College has 
moved on to a more team based Caseloading model, or has abandoned workloading altogether.

\section{Caseloading}

The Caseloading model is one that usually encompasses teams, rather than individuals. In this model the team is responsible for managing a caseload of students, linked to their recruitment targets, and achieving a set level of outcomes, usually qualification outcomes. They are given the responsibility for delivering these outcomes within the resources generated by the student numbers. The team can deploy the resources in a flexible way, to best achieve the outcomes and meet the needs of their specific students. The individual workload and balance of team members may differ and they may choose to specialise in activities or diversify. They may also decide on the learning styles to adopt and the types of staff that should be employed on the programme. The ultimate Caseloading model devolves the budget for curriculum delivery to the team, for staff, consumables and equipment. The staff then plan how to deploy those resources to achieve the planned outcomes. The manager's role is to monitor activities and ensure budgets are contained and targets met. Development activities become the responsibility of the team and they are free to release individuals from teaching to undertake other activities that the team see as necessary. Annual teaching loads are not considered in a Caseloading model, as the team allocates activities to members to meet the curriculum and development needs. Sallis (1996) 
identifies the benefits of a Caseloading model in assuring quality and outlines the devolved model as one where,

"... it is the teaching unit's decision as to how much resource is put into direct teaching, projects, tutorials, workshops, resource-based learning or any other approach to learning. Academic units are given the freedom to adopt new and innovative approaches if they feel it is to the students' benefit. They can decide on the workloads of their staff and distribute work in ways which meet their curriculum goals rather than have staff workloads determined by senior management." (p92)

Control of resources therefore passes to teams, rather than remaining with managers. This has potentially far reaching implications for the roles of all staff involved in Caseloading. In reality the model of Caseloading adopted is along a continuum of autonomy which gives more or less responsibility to the teams involved.

\section{Pressure to Change}

The terms, conditions and working arrangements of teaching staff in Further Education have continued as the subject of debate since the 1980's. Following Incorporation in 1993 this debate gathered speed, as the separate

colleges became independent employers, each with their own agenda and preferred ways of working. Caseloading therefore came to the fore as an 
issue following Incorporation, as a potential method for changing ways of working..

Caseloading was at the outset intended to be a method for changing working practices, at least by the employers. A number of pressures are identified within the Further Education sector which give rise to interest in Caseloading. These include; the move from teaching to learning, more student centred approaches, the changing role of the teacher, flexible employment contracts and the rate of change within the sector. The impetus for Caseloading is identified in Kedney \& Scribbins (1995a) report as coming from the pressure for greater flexibility. This is flagged as an industrial relations issue. Resource management, to create efficiency gains, including spreading activity throughout the year is a second driver towards Caseloading methods. A cultural change towards control of resources at delivery level is identified as the third driver for change. Kedney \& Scribbins highlight the support within the sector for the cultural change aspects and the empowerment objective as a long-term aspiration in using Caseloading models.

To date the search for one key model, which encompasses Caseloading for the whole sector, has failed, as Kedney and Scribbins note, Caseloading models are very context specific.

Caseloading was generated in part as a mechanism for coping with the cultural changes being forced on Further Education. Gorringe (1994) highlights this in his discussion of the move from an "allocation" to an 
"earning" model of funding. Bush and West Burnham (1994) also identify output budgeting as a rational model, rather than one that focuses on inputs. By this method resource allocation can be directly related to achieving the objectives of the organisation. Gorringe (1994) points out the change from a Local Authority model which always seemed to be a struggle to get more money, to one based on earning money based on delivery of high quality services. He identifies that the emphasis has therefore changed from a model based on competency, to one that takes a holistic view of delivery of services. Hours of work, holidays, and reward schemes all need to reflect this change. This shift in resource allocation model creates a greater need for systematic planning because resources must be related to objectives in advance. The objectives themselves must also be specified in a systematic way. In addition, priorities are established, because alternatives will be available, and performance indicators are necessary to assess the effectiveness of the use of resources and allocation of future resources.

Why has Caseloading not been widely adopted?

Pressure on working practices continues well after Incorporation, despite the warning given by Leevers and Dixon (1999) that, 
"Effort should be concentrated on improving the student rather than the teacher - the quality of the learning experience rather than the issues such as contact hours or contracts... " (p8)

Pressures to change working practices within Further Education have been hard to resist since 1993. They have come in the form of funding methodologies, which direct effort and rewards in specific directions, curriculum changes, legislative changes, and re-drafting of the remit for governors. New initiatives have been introduced which require new ways of working and colleges have been expected to rise to the challenge. Caseloading was designed as one way of responding to these pressures. It has a dual objective; the first is of devolving authority, responsibility and control to delivery teams, locating the decision making process closer to the client base. This fits in with latest management theories on motivation through empowerment and professional control of tasks. It also hits the spot politically as accountability in public life becomes increasingly emphasised. Public organisations must account for how they spend their money. They must also be seen to spend it wisely. Organisations are being held accountable, and they in turn are holding individual managers accountable. Devolution (itself a current buzzword) makes managers accountable for their actions and the budgets they manage. They receive devolved blame as well as power. Hopefully they receive devolved praise and credit too.

The second objective for the introduction of a Caseloading model is to reduce costs, create flexible deployment of staff and remove what have been seen as 
restrictive working practices. Since 1993 employers have worked to change teacher contracts, this has to a large extent been achieved, although many feel there are yet more changes required. Nationally the emphasis on reinstating the national employment framework has officially gone and local negotiations are seen as the best response to local situations.

Major changes to working practices have been achieved, specifically with the introduction of teaching support roles in curriculum delivery. These posts are on different terms and conditions to teaching staff, and often present a more cost-effective way of delivering standard programmes. Kedney and Scribbins (1995) did the initial work on the introduction of Caseloading in Further Education and their reports have been discussed earlier. Kedney also published details of the use of teaching support staff in 1999. The report identifies that in the 112 colleges reviewed in 19982,178 full time equivalent teaching support posts were being used compared with 854 in 1996 . This almost three fold increase highlights the changes in curriculum delivery that have occurred within the sector. The majority of these posts are on Business Support contracts; not teaching contracts and are in practical rather than "academic" curriculum areas. The survey results identify the intention to increase the use of such posts in the future. A parallel trend is identified in the growing use of fractional posts. This is largely in response to the change in employment legislation, which removes the flexibility of contracting for sessional staff and increases the employment costs. Changes to working methods have therefore been achieved in many instances without the use of Caseloading. These changes have potentially wide ranging implications for 
the roles and future employment of lecturers, as lecturing posts are replaced by teaching support posts.

The secondary motives of devolution of budgets to create team empowerment and motivation also seems to be being sidetracked. As Leevers and Dixon (1999) identify few colleges who are achieving reduced costs of delivery as well as high quality provision are using devolved budgets. Centralisation of resources allows for greater control and monitoring and may reduce bureaucracy and costs by removing repetitious systems. It also reduces the need for sophisticated management information systems to generate cost centre data.

The weakness of the management information systems used by many colleges is central to the failure to adopt the Caseloading model. Colleges continue to struggle to produce accurate student and staff statistics in form acceptable to the FEFC and useful to the organisation. With this level of difficulty being experienced in producing accurate, timely and useful information, there is little wonder that a Caseloading model which requires sophisticated MIS systems to devolve resources based on income generated to teams has found little favour. Serious difficulties have been identified with the information systems and investment made in new software systems and hardware across the sector. Effective register, timetabling, payroll and finance systems are required which create an integrated management information system which managers can trust to provide data with which to manage devolved budgets. Major investment in monetary terms plus the 
organisational backing to make the systems work is required. With consideration being given to the future funding methodology under the Learning and Skills Council this investment is unlikely as the data reporting requirements may well change.

A lack of a common framework and a confusion over the terminology about Caseloading has clouded the issue I feel and detracted from moves to introduce it. The common conception of the system as being one of weighted workload measurement creates resistance from managers and suspicion from teachers. This system, although used in a number of colleges, creates a large administrative burden, purely in the establishment of the weightings, managing their implementation and tracking the staff utilisation. This model is unlikely to create costs savings without radical changes in the staff profile and the introduction of a major element of teaching support, to remove the dependence on lecturers. Creating weightings for activities may produce equity of workload, but it is likely to increase costs, as who is going to accept an increase in workload? All weightings will start from the lowest common denominator base and additional weighting be granted to all other work. An increase in delivery costs is therefore the most likely outcome of this type of model.

Haythornewaite (1996) identifies a number of organisational factors that must be available to make devolved budget models such as Caseloading work. These include leadership on principles and practices to be used; on the skills and techniques of team and budget management. Central management must 
be committed to the objectives underlying the devolution and communicate a clear understanding of these objectives. Middle managers, who will be managing the budgets, must be involved and the understanding of the objectives must be promoted. Continuing training in management techniques and the provision of adequate manuals and information underpin success. On the opposite side, sanctions against budget holders who do not manage their budgets, or achieve their targets must be negotiated and agreed. In a time of reduced resources and increased workload Caseloading models and the training to support them are rarely given priority.

Simkins (1989) identifies some of the internal political pressures that affect budgeting processes and the authority within the subgroups of an organisation. He also stresses the strategic leadership aspects of the system and the central role that organisational leaders play in establishing the budgetary style. Differing views on Caseloading and what it could and should achieve can block the successful introduction of the model. Organisational changes in contracts, terms and conditions as well as curriculum delivery make some of the looked for changes unnecessary, or achieved them in other ways.

As a concept the devolved Caseloading model can work to create empowered teams. With the right level of information and organisational support I feel that the model has many strengths. Not least of these is the authority that it gives to teams and managers over the deployment of resources, including their own time. With the right level of involvement at all levels in target setting, 
transparent systems for allocating resources linked to activity, regular and accurate information on costs, expenditure and outputs, a devolved system can empower, enthuse and motivate teams. A culture of innovation and development can be fostered and resources utilised in a way, which best meet client needs. Organisational commitment in terms of investment in systems, training and support for implementation is vital. Real belief in the system must be exhibited from the top, and trust built up that the devolved control will not be arbitrarily removed when circumstances change.

I feel that Caseloading can provide a more effective management model, but not without clear and detailed management commitment. As to whether it is the best way of achieving its aims, I feel it is not, as a management model it has been has been overtaken by events and other more appropriate methods are available to achieve the objectives, responding to the changing environment of Further education. 


\section{References}

Association of Colleges A national survey of current practice: Assistant lecturers, Instructors, Supervisers and Associated posts circular 21/99 7 June 1999

Bush T \& West-Burnham J (1994) Principles of Educational Management Harlow UK: Longman

Carrol S \& contributors (1996), Caseloading

FEDA Report Developing Further Education series. London: FEDA

Gorringe R, Bridge W, Silver R, Henry T, Limb A, Ruddiman K, Lewis N, Togher J, (1994) Changing the culture of a college Coombe Lodge Report vol. 24 no. 3 Bristol: The Staff College

Haythornewaite et al (1996) A Guide to Devolved Budgeting London: CIMA

Kedney B, Scribbins K (1995a) Case-loading in Colleges

Joint Review Group Report Occasional Paper 95/1 London: CEF/APC

Kedney B, Scribbins K (1995b) Case-loading in Action

Joint Review Group Report Occasional Paper 95/5 London: CEF/APC 
Kedney B, Scribbins K (1995c) Register of Caseloading in Colleges Joint Review Group Report Occasional Paper 95/6 London: CEF/APC

Leevers S, Dixon S (1999) More and better for less: a three-card trick FEDA Bulletin Vol. 2 No. 12 London: FEDA

Peeke G (1999) Changing College Cultures

FEDA Report Vol. 2 No 8 London: FEDA

Simkins T (1989) Budgeting as a political and organizational process, in

Levacic R (ed) Financial Management in Education

Milton Keynes: Open University Press 